component of chronic kidney disease, we investigated whether the associations between decline in eGFR and the outcomes differ based on individuals' baseline blood pressure. Results: In the age and sex adjusted models, each unit $\left(\mathrm{mL} / \mathrm{min}\right.$ per $\left.1.73 \mathrm{~m}^{2}\right)$ annual decline in eGFR was associated with higher occurrence of subcortical infarcts (OR: $1.11 ; 95 \% \mathrm{CI}: 1.03,1.21$ ) and $2 \%$ higher white matter hyperintensity volume progression (95\%CI: 1.06, 2.87). There was no association between decline in kidney function and occurrence of new cortical infarcts or microbleeds. Further adjustments for cardiovascular risk factors did not change the findings. Stratifying for baseline blood pressure, participants with higher systolic and diastolic blood pressure had higher occurrence of new MRI-detected infarcts per units of eGFR decline ( $\mathrm{P}$ for interaction 0.008 and 0.031 , respectively).We observed a suggestive difference in the association of eGFR decline with white matter hyperintensity volume progression in different levels of systolic blood pressure, indicating that participants with lower systolic blood pressure are more likely to have white matter hyperintensity volume progression as their eGFR declines (P for interaction 0.096) (Figure 1). Conclusions: Our findings suggest that older individuals with steeper decline in kidney function are at an increased risk for development of subcortical infarcts and white matter hyperintensities and should be considered as high risk populations for developing accelerated subclinical brain vascular pathologies.

\section{O1-02-03 POST-TRAUMATIC STRESS DISORDER AND RISK OF DEMENTIA AMONG MEN AND WOMEN MEMBERS OF A HEALTHCARE DELIVERY SYSTEM}

Jason Flatt ${ }^{1}$, Charles P. Quesenberry, Jr, ${ }^{2}$, Jennifer Y. Liu ${ }^{2}$, Kathleen Albers ${ }^{2}$, Rachel A. Whitmer ${ }^{2},{ }^{1}$ University of California, San Francisco, San Francisco, CA, USA; ${ }^{2}$ Kaiser Permanente Division of Research, Oakland, CA, USA. Contact e-mail: jason.flatt@ucsf.edu

Background: Past studies with predominately male cohorts have shown an increased risk of dementia in those diagnosed with post-traumatic stress disorder (PTSD). While research suggests that women may develop PTSD more often than men, little is known about PTSD and risk of dementia in women. We examined risk of dementia and association with PTSD over 13 years among both older men and women who were members of a healthcare delivery system. Methods: We examined 499,844 patients of Kaiser Permanente Northern California medical system who were age $\geq 60$ years as of January 1, 2002. Dementia diagnoses were collected from medical records January 1, 2002-December 31, 2014, with a mean follow-up time of 8.2 years. Baseline risk factors including diagnoses of PTSD, diabetes and stroke were abstracted from medical records from January 1, 1996 to December 31, 2001. We evaluated associations of PTSD and dementia risk using multivariable cox proportional hazards models stratified by gender and adjusted for age, race, diabetes and stroke, with censoring for death and $>90$ day gaps in health plan coverage. Results: The mean age at baseline was 71.0 years (range 60-102), with $54.7 \%$ female, $68.0 \%$ White, 5.5\% Black, $6.5 \%$ Hispanic, 9.0\% Asian, and 1,147 patients were diagnosed with PTSD. Over the 13 years of follow-up, $11.8 \%(59,127)$ were diagnosed with dementia with an average follow-up time of 8 years. Those with PTSD were 56\% more likely to develop incident dementia compared with those without PTSD (adjusted Hazard Ratio [HR] $=1.56$, 95\% Confidence Interval $[\mathrm{CI}]=1.33,1.83$ ). Gender stratified models (Table 1) showed
Table 1

Association between post-traumatic stress disorder (PTSD) and risk of dementia in multivariable-adjusted models stratified by gender.

\begin{tabular}{llll}
\hline & All patients & Men & Women \\
\cline { 2 - 4 } Model & HR $(95 \% \mathrm{CI})$ & & \\
\hline Demographics & $1.58(1.34-1.85)$ & $1.96(1.49-2.52)$ & $1.42(1.15-1.73)$ \\
$\begin{array}{l}\text { Diabetes and } \\
\quad \text { Stroke }\end{array}$ & $1.56(1.33-1.83)$ & $1.89(1.44-2.43)$ & $1.41(1.14-1.72)$ \\
\hline
\end{tabular}

that men with PTSD had a $90 \%$ increase in dementia risk and women with PTSD a $41 \%$ increase compared to those without PTSD (men: $\mathrm{HR}=1.89, \mathrm{CI}=1.44,2.43$; women: $\mathrm{HR}=1.41$, $\mathrm{CI}=1.14,1.72$ ). Conclusions: This is the first large study of PTSD and risk of dementia in women and results suggest that PTSD is a risk factor in both genders. Future studies are needed to better understand the mechanisms linking PTSD and dementia as well as potential gender differences. Future efforts aimed at screening and improving treatment outcomes in both men and women with PTSD should be considered.

\section{O1-02-04 PRIOR TRAUMATIC BRAIN INJURY IS ASSOCIATED WITH SUBJECTIVE BUT NOT OBJECTIVE MEMORY IMPAIRMENT AMONG NON-DEMENTED OLDER ADULTS IN THE HEALTH AND RETIREMENT STUDY}

Raquel C. Gardner ${ }^{1}$, Kenneth M. Langa ${ }^{2}$, Kristine Yaffe ${ }^{1},{ }^{1}$ University of California San Francisco / San Francisco VA Medical Center, San

Francisco, CA, USA; ${ }^{2}$ University of Michigan, Ann Arbor, MI, USA. Contacte-mail: raquel.gardner@ucsf.edu

Background: Traumatic brain injury (TBI) has been implicated as a risk factor for Alzheimer's disease (AD). Pre-clinical features of AD may include mild memory and affective symptoms. Our aim was to determine whether prior TBI is associated with worse memory function or depression among non-demented older adults, as chronic effects of TBI in this population are understudied. Methods: We studied 1,270 non-demented older adults randomly selected from self-respondents to the 2014 wave of the Health and Retirement Study for a detailed assessment of lifetime history of TBI. TBI was defined as prior head injury leading to loss of consciousness, post-traumatic amnesia, or hospitalization. Four outcomes were assessed: objective memory impairment ( $\geq 1$ standard deviation below the cohort mean on a 10 -word delayed free-recall task), subjective memory impairment (self-rating of memory as "fair" or "poor" on a 5-level scale: excellent, very good, good, fair, poor), active depression (score of $\geq 3$ on the 8 -item Center for Epidemiologic Studies Depression Scale), and self-reported history of physician-diagnosed depression. Risk of memory and depression outcomes were compared between groups using regression models adjusting for age, gender, race, ethnicity, education and medical comorbidities. Results: TBI participants $(n=529)$ were younger (mean age $65 \pm 8$ vs. $68 \pm 10$ years), less likely to be female or Hispanic, and had higher prevalence of chronic lung disease, heart disease, and arthritis compared to no-TBI participants $(n=741)$. Mean time since most recent TBI was $34 \pm 19$ years. In adjusted models, TBI participants had higher risk of subjective memory impairment (32\% vs. $27 \%$; risk ratio [RR] $1.21,95 \%$ confidence-interval [CI] 1.02-1.44), active depression (22\% vs. $14 \%$; RR 1.49 , 95\% CI 1.17-1.91), and physician-diagnosed depression (32\% vs. $20 \%$; RR $1.37,95 \%$ 
CI 1.13-1.66) compared to no-TBI participants. Risk of objective memory impairment, however, was not increased among TBI participants (14\% vs. 18\%; RR 0.93, 95\% CI 0.72-1.20). Conclusions: Non-demented older adults with prior TBI were more likely to report subjective memory impairment, active depressive symptoms, and physician-diagnosed depression. Lack of objective memory impairment may be due to poor sensitivity of the memory instrument, subjective-only memory impairment (possibly depression-related), survival bias, or suggests that post-TBI cognitive impairment primarily affects non-memory domains.

\section{O1-02-05 PRESBYCUSIS AND DEMENTIA: RESULTS FROM 8 YEARS OF FOLLOW-UP IN THE THREE-CITY MONTPELLIER STUDY}

Arthur Meusy $^{1,2}$, Audrey Gabelle ${ }^{3,4}$, Laure Anne Gutierrez ${ }^{1,3}$, JeanLuc Puel $^{5,6}$, Frederic Venail ${ }^{5,7}$, Claudine Berr ${ }^{1,3,6},{ }^{1}$ INSERM U1061, Montpellier, France; ${ }^{2}$ CHRU Montpellier, Montpellier, France; ${ }^{3}$ Memory Resources and Research Center, CHRU Gui de Chauliac, Montpellier, France; ${ }^{4}$ INSERM-UM 1183, Montpellier, France; ${ }^{5}$ INSERM UMR 1051, Montpellier, France; ${ }^{6}$ University of Montpellier, Montpellier, France; ${ }^{7}$ CHRU Montpellier, Montpellier, France. Contact e-mail: claudine.berr@ inserm.fr

Background: Presbycusis (age related hearing impairment) is a potentially modifiable risk factor or an early marker of dementia. Several pathways may explain the link between hearing loss and dementia: common vascular factors, frailty, social isolation or a change of the cognitive load. Our objective was to assess the association between presbycusis, evaluated with pure-tone audiometry, and incident dementia, in a population based cohort of elderly subjects. Methods: Subjects from the Three-City study (Montpellier center), who underwent a tonal audiometry and were dementia-free at this baseline, were followed from 2003 to 2012 every two to three years. Presbycusis was defined as a decrease in Pure Tone Average (PTA) greater than $25 \mathrm{~dB}$ on the better hearing ear in subjects with no earrelated diseases. All dementia cases diagnosed by the E-3C neurologist were reviewed by an independent committee of neurologists. Multivariate Cox proportional hazards models were used to assess the link between presbycusis at baseline and incident dementia, controlling for age, sex, education, living alone, Apo E4, diabetes mellitus, obesity, smoking, hypertension, ischemic heart disease, depression, subjective health status, number of medications taken and instrumental activities of daily living. Results: 582 individuals (median age 74.6, 60\% of women) were followed for a median of 95 months. During this period we observed 35 incident dementia cases. $77 \%$ of them had presbycusis at baseline compared to $46 \%$ in subjects free of dementia. Risk of dementia was increased in subjects with presbycusis when controlling for age (hazard ratio $(\mathrm{HR})=$ 3.22 , IC 95\% [1.44; 7.22], $\mathrm{p}=0.01)$. It remained significant when controlling for various demographic, lifestyle, vascular and health factors $(H R=3.13$ [1.34; 7.28], $\mathrm{p}=0.01)$. Conclusions: Incident dementia is independently associated with presbycusis in this aged cohort. Our data did not allow us to study the effect of hearing aids on this association. But we can suggest that timely presbycusis diagnosis with appropriate hearing aid might delay dementia onset.

\section{O1-02-06 PLASMA CLUSTERIN IS ASSOCIATED WITH AD- VULNERABLE BRAIN REGIONS IN MIDDLE-AGE ADULTS: THE CARDIA BRAIN MRI STUDY}

Thaddeus J. Haight ${ }^{1}$, R. N. Bryan ${ }^{2}$, Russell Tracy ${ }^{3}$, Myriam Fornage ${ }^{4}$, Osorio Meirelles ${ }^{1}$, Melissa Richard ${ }^{4}$, Christos Davatzikos ${ }^{2}$, Ilya Nasrallah ${ }^{5}$, David R. Jacobs, Jr, ${ }^{6}$, Cora Lewis ${ }^{7}$, Pamela Schreiner ${ }^{6}$, Stephen Sidney ${ }^{8}$,
Lenore J. Launer ${ }^{1},{ }^{1}$ National Institute on Aging, Bethesda, MD, USA; ${ }^{2}$ Center for Biomedical Image Computing and Analytics / University of Pennsylvania, Philadelphia, PA, USA; ${ }^{3}$ University of Vermont, Colchester, VT, USA $;{ }^{4}$ University of Texas Health Science Center at Houston, Houston, TX, USA; ${ }^{5}$ University of Pennsylvania, Philadelphia, PA, USA; ${ }^{6}$ University of Minnesota, Minneapolis, MN, USA; ${ }^{7}$ University of Alabama, Birmingham, AL, USA; ${ }^{8}$ Kaiser Permanente, Oakland, CA, USA. Contact e-mail:tad.haight@nih.gov

Background: A gene regulating clusterin has been shown to increase the risk for Alzheimer's disease (AD). Studies of older persons with higher plasma clusterin (PC) have reported increased prevalence of $\mathrm{AD}$ and faster clinical progression. In the context of identifying early biomarkers of risk, it is of interest to investigate the association of $\mathrm{PC}$ with characteristics of $\mathrm{AD}$, which include vulnerable brain regions, in middle age individuals from the community. Methods: Subjects were 639 cognitively normal individuals (mean age $50 \pm 3.5 \mathrm{SD}$ ) from the Coronary Artery Risk Development in Young Adults (CARDIA) Brain MRI Study. PC (mean 255 $\pm 31 \mathrm{ng} / \mathrm{ml}$ ) was quantified with sandwich ELISA using samples prepared by standard protocol. PC was investigated in association with brain regions affected early in $\mathrm{AD}$, as described by Braak staging: entorhinal cortex (ECV), hippocampus (HV), and medial temporal lobe (MTLV) volumes $\left(\mathrm{cm}^{3}\right)$. Total brain volume (TBV) and larger brain structures affected in later Braak staging were examined for comparison. Preliminary analyses suggested curvilinear relationships between PC and volumes of the smaller structures (Figure 1), therefore, we fit quadratic models to assess their associations, adjusting for age, sex, race, intracranial volume, C-reactive protein, diabetes, and dyslipidemia. Results: Higher PC had a negative non-linear association with lower ECV (combined left and right side), as indicated by Figure 1 which documents the quadratic model fit and concordance with decile means, that was influenced by highest PC levels $(\mathrm{p}<0.025)$, adjusted for covariates. Similar relationships were observed between PC and $\mathrm{HV}$, although the relationship was stronger for the left-side HV than the right-side. After adjustment, the association was non-significant. Associations between PC and MTLV were strongest for the left-side: higher PC was associated with lower MTLV, and this negative non-linear association was influenced by highest PC levels $(\mathrm{p}<0.045)$, adjusted

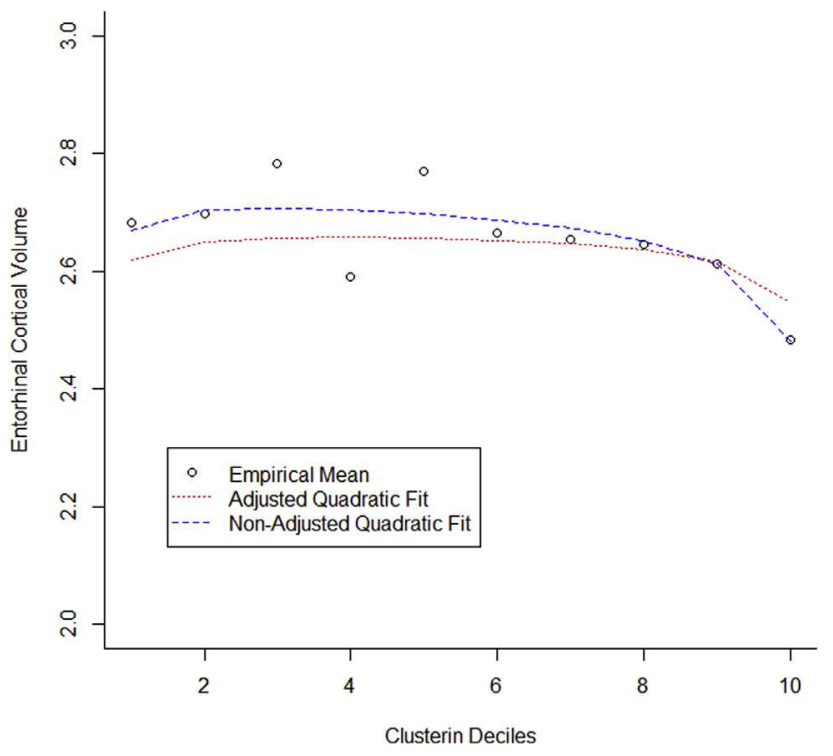

